\title{
BMJ Open The 'Hikikomori' syndrome: worldwide prevalence and co-occurring major psychiatric disorders: a systematic review and meta-analysis protocol
}

\author{
Andrea Pozza, ${ }^{1}$ Anna Coluccia, ${ }^{1}$ Takahiro Kato, ${ }^{2}$ Marco Gaetani, ${ }^{1}$ Fabio Ferretti ${ }^{1}$
}

To cite: Pozza A, Coluccia A, Kato T, et al. The 'Hikikomori' syndrome: worldwide prevalence and co-occurring major psychiatric disorders: a systematic review and metaanalysis protocol. BMJ Open 2019;9:e025213. doi:10.1136/ bmjopen-2018-025213

- Prepublication history and additional material for this paper are available online. To view these files, please visit the journal online (http://dx.doi. org/10.1136/bmjopen-2018025213).

Received 04 July 2018 Revised 02 September 2019 Accepted 05 September 2019

Check for updates

(c) Author(s) (or their employer(s)) 2019. Re-use permitted under CC BY-NC. No commercial re-use. See rights and permissions. Published by BMJ.

${ }^{1}$ Department of Medical Sciences, Surgery and Neurosciences, Santa Maria alle Scotte University Hospital of Siena, Siena, Italy

${ }^{2}$ Department of Neuropsychiatry, Graduate School of Medical Sciences, Kyushu University, Fukuoka, Japan

Correspondence to Professor Fabio Ferretti; ferrefa@unisi.it

\section{ABSTRACT}

Introduction The 'Hikikomori' syndrome (HS) consists of prolonged and severe social withdrawal. It has been studied first in Japan and recently has increasingly drawn the attention of researchers and clinicians all over the world. It is unclear whether it exists in other cultural contexts than Asia. The existing systematic reviews did not provide a quantitative synthesis on its prevalence. In addition, a summary of the co-occurring rates of psychiatric disorders is lacking. To provide a more comprehensive understanding of the clinical picture, it seems important to investigate which psychiatric disorders listed in the classification systems are most frequently associated with this psychological condition affecting young people. This paper describes a systematic review and meta-analysis protocol summarising worldwide prevalence of the HS in general population and clinical samples with psychiatric disorders. The review will also assess the co-occurrence between HS and each psychiatric disorder defined by any version of the Diagnostic and Statistical Manual of Mental Disorders (DSM) or International Classification of Diseases (ICD) in any clinical samples with psychiatric disorders.

Methods and analysis A systematic review will be conducted according to Preferred Reporting Items for Systematic Reviews and Meta-analyses (PRISMA) guidelines. Studies will be included if they use youth aged 12-35 years, recruited from general population or population with psychiatric disorders, if they use international criteria to diagnose HS. No restriction about design or language will be applied. The search will be conducted during the first week of November 2019 by two independent reviewers through the databases Scopus, PubMed, PsycINFO, Web of Science, by examining study references, by looking for conference proceedings/ dissertations/theses, by contacting study corresponding authors. Random-effect meta-analysis will be performed by computing effect sizes as logit event rates. Study quality will be assessed through the Newcastle-0ttawa Scale.

Ethics and dissemination The current review does not require ethics approval. The results will be disseminated through conference presentations and publications in peer-reviewed journals.

PROSPERO registration number CRD 42018098747.
Strengths and limitations of this study

- The most recent review conducted the literature search in February 2015 and did not analyse prevalence rates.

- There is a lack of a systematic review with meta-analysis summarising prevalence of the Hikikomori syndrome (HS) in general population and in psychiatric population.

- Our review is the first study exploring the co-occurrence between HS and psychiatric disorders.

- Potential limitations will be a small number of the studies to be included or the heterogeneity across the studies in the criteria used to define HS or in the measures to assess it.

\section{INTRODUCTION}

The term 'Hikikomori' derives from Japanese, and it is composed of the verb 'hiki (hiku)', which means to move back, and 'komori (komoru)', which means to come into. ${ }^{12}$ In the last two decades, the 'Hikikomori' syndrome (HS) has been conceptualised as a psycho-sociological condition characterised by prolonged and severe social withdrawal for a time period of at least 6 months. ${ }^{3-5}$ This condition has been reported and studied first in the Japanese society/ culture. ${ }^{6}$ In the first epidemiological research conducted in 2003, ${ }^{78}$ the Japanese Ministry of Health, Labour and Welfare defined it as a state in which a young individual (a) mainly stays at home, (b) cannot or does not engage in social activities, such as going to school or working, (c) has continued in this state for 6 months or longer, (d) has neither a psychotic disorder nor a medium to lower level of mental retardation (IQ <55-50) and (e) has no close friends.

Social withdrawal in the HS typically involves staying at home almost all days. ${ }^{69}$ Some authors have proposed two subtypes of social withdrawal behaviour characterising 
the HS: the 'hard core' subtype, including those youths who never leave their room and never talk to their families and the 'soft' subtype, including those cases who go out and talk to others occasionally. ${ }^{10}$ More recently, Kato and colleagues ${ }^{11}$ have proposed another subtyping of the HS, distinguishing between those cases who live with their families-this group represents the majority of the HS population-and those who live alone, representing about $11 \%$. Typically, severe social withdrawal behaviour affects males (4:1 male-to-female ratio), mostly the young adult eldest son of a family with a good socioeconomic and cultural level. Age of onset can vary from 20 to 27 years, but prodromal symptoms often emerge during early adolescence. ${ }^{68}$

It has been hypothesised that some of the socially withdrawn youths have close friends but do not maintain contact with them during social withdrawal or that do not have any close friends but maintain alternative, less-demanding personal relationships with others such as online friends. ${ }^{12}$ Socio-cultural influences have been believed as key factors involved in the development of this condition, such that some authors have proposed the inclusion in the DSM-5 'culture-bound' syndromes chapter as a Japanese syndrome. ${ }^{13}$ The role of cultural aspects was supported in other psychiatric disorders which similarly to the HS share social withdrawal as a key component or maintenance factors such as psychotic disorders, social anxiety disorder, depressive disorders, obsessive-compulsive disorder, and Internet addiction. ${ }^{14-20}$ Other researchers and clinicians believe that this form of social withdrawal behaviour is only a symptom of a wide variety of major psychiatric disorders listed in DSM-IV and the current DSM-5 (eg, psychotic disorders, depressive disorders, social anxiety disorder, agoraphobia, schizoid or avoidant personality disorder, Internet addiction).$^{21}$ Consistent with the latter hypothesis, the concept of 'secondary Hikikomori' has been proposed to define those cases whose severe social withdrawal behaviour is a manifestation of a subtype of another psychiatric disorder or even a consequence of a primary psychiatric disorder. ${ }^{12}$ Other authors suggest that a considerable subset of the cases present with clinical features that do not meet the criteria for any of the existing psychiatric disorders ${ }^{13}$; hence, it has been suggested that the HS could be considered as a primary new psychiatric disorder in a future version of the DSM, despite having some clinical overlap with other disorders. ${ }^{22}$ Consistent with this hypothesis, Kondo and colleagues $^{23}$ reported that in a group of patients aged 16-35 years with onset of social isolation before age 30 for at least 6 months, $8 \%$ had schizophrenia, $26 \%$ had an anxiety disorder, $8 \%$ had a depressive disorder, $23 \%$ had a personality disorder (including 6 with Avoidant, 6 with Schizoid, and 4 with obsessive-compulsive personality disorder).

As recently summarised by Kato $e t a l,{ }^{24}$ at the present time whether it is other psychiatric disorders that give rise to hikikomori as a symptom or whether it is indeed HS that is the cause of co-occurring major mental health conditions has not been clearly established; thus, it could be argued that both possibilities exist. The authors identified a group of psychiatric disorders characterised by hikikomori-like features including psychosis, social anxiety disorder, avoidant personality disorder, depressive disorders, Internet addiction, and post-traumatic stress disorder. ${ }^{24}$ Such disorders would be the most frequently co-occurring ones with HS.

Other researchers suggested that the HS might not be a culture-bound syndrome depending on the socio-cultural context but that it may exist also outside Asian countries. ${ }^{22}$ Epidemiological research conducted on community general population has produced quite heterogenous prevalence data, showing that the prevalence of the HS can range from approximately $0.87 \%{ }^{25}$ to $1.2 \%$ in Japan, ${ }^{26}$ to $1.9 \%$ in Hong $\mathrm{Kong}^{27}$ to $2.3 \%$ in Korea $^{28}$ or up to $26.66 \%$ in student population in Japan. ${ }^{29}$ This variety may depend on differences in the inclusion criteria, assessment instruments, studies' countries, and recruitment strategies across the studies. Research conducted in clinical samples with psychiatric disorders or in treatment-seeking population in mental health services demonstrates that the prevalence can vary from $12.64 \%^{30}$ up to $63.07 \% .{ }^{28}$ The socio-cultural features of the HS probably involve a variety of cross-cultural factors such as the social structure (eg, the mainstreaming culture, the labelling effects, the academic expectations imposed to students which are prominent in Asian countries but also in other countries), the society's media (eg, media enunciation when reporting the issue), the school context (eg, the bullying phenomenon), and the family relationships (eg, enmeshed parent-child relationships). ${ }^{115182022}$

In addition, one of most important problems related to studying this condition involves the heterogeneity in the definitions used across the studies and a lack of consensus on well-established diagnostic criteria. ${ }^{12}{ }^{31}$ For example, some studies conducted in Japan have considered a duration of severe social withdrawal that is longer than 6 months as a clinically meaningful threshold, while other research conducted in Korea ${ }^{28}$ and Hong Kong ${ }^{32}$ has used a shorter duration criterion (3 months). Recently, Teo and Gaw ${ }^{13}$ conducted an online and manual systematic search of the HS criteria using the Pubmed and PsycINFO databases. The researchers provided a proposal for diagnostic criteria based on the most recurrent clinical features and defined the HS as (a) spending most of the day and almost every day at home, (b) marked and persistent avoidance of social situations, (c) the social withdrawal and avoidance interferes significantly with the person's normal routine, occupational (or academic) functioning, or social activities or relationships, (d) the person perceives the withdrawal as ego-syntonic, (e) in individuals under age 18 years, the duration is at least 6 months, (f) the social withdrawal is not better accounted for by another mental disorder (eg, social anxiety disorder, major depressive disorder, schizophrenia, or avoidant personality disorder). ${ }^{133}$ 
In the last decade, some reviews have been conducted on the HS $^{32}{ }^{34-36}$; however, only one study ${ }^{12}$ used well-established guidelines for systematic reviews (ie, Preferred Reporting Items for Systematic Reviews and Meta-analyses (PRISMA) criteria). ${ }^{37}$ Recently, Li and Wong ${ }^{12}$ conducted a systematic review of 42 qualitative and quantitative studies by searching online databases (ProQuest, ScienceDirect, Web of Science, PubMed). The authors identified 12 qualitative studies using case study designs, focus group or ethnographic research methods, 9 expert opinion papers and 3 reviews. ${ }^{12}$ In addition, 19 quantitative studies were identified: however, 3 out of them used a case series design. Out of the quantitative studies, 10 were conducted in Japan, 3 in China and 1 in Korea, thus confirming partially the socio-cultural roots of the phenomenon but also highlighting the need for research on contexts different from Asian countries. ${ }^{12}$

\section{Rationale for this study}

The HS has increasingly drawn the attention of researchers and clinicians in the last two decades ${ }^{13} 38$; however, most research included anecdotal reports or single case studies, and few contributions used a quantitative methodology. ${ }^{22}{ }^{38}{ }^{39}$ Despite the first reports of the HS were conducted first in Japan or in other Asian contexts, it is unclear whether the phenomenon may exist in other cultural contexts such as European and American countries. Further knowledge is needed, since the existing systematic review ${ }^{12}$ searched papers in February 2015, and it did not provide a quantitative summary of the prevalence rates of the HS using meta-analysis pooling data from primary studies. If a variability in the worldwide prevalence is present, it seems important to explore the role of socio-demographic and cultural factors (ie, type of country) in this variability for a better understanding of this phenomenon. Moreover, a systematic review with meta-analysis summarising the prevalence rates of this condition and the co-occurrence of psychiatric disorders has not been conducted. None of the previous reviews summarised the co-occurring rates of psychiatric disorders in HS. In order to have a more comprehensive understanding of the clinical picture of this condition, it seems important to investigate which psychiatric disorders are most frequently associated with it.

\section{Objectives}

This paper describes the protocol for a systematic review and meta-analysis study of primary observational studies, to provide a quantitative synthesis on the worldwide prevalence rates of the HS and the co-occurrence rates of major psychiatric disorders. Specifically, the aims will be: (1) to investigate the prevalence rates of the HS in general population (general population will include individuals recruited from community or students/undergraduates); (2) to investigate the prevalence rates of the HS in treatment-seeking clinical samples with psychiatric disorders listed in classification systems, recruited from primary, secondary or tertiary mental health settings; (3) if significant heterogeneity is found, age, gender and type of countries where the study has been conducted (Asian vs non-Asian countries) will be investigated as potential moderators of the prevalence rates in both the general population and the clinical samples with psychiatric disorders; (4) to assess the co-occurrence between HS and each psychiatric disorder defined by the criteria of any version of DSM or ICD in any clinical samples with psychiatric disorders. To provide a broad overview of the co-occurrence between HS and psychopathology, we will consider any psychiatric disorder based on any version of these two classification systems which are the most internationally used systems (see $\S$ Data extraction and coding procedure). Following the clinical observations and hypotheses proposed by previous authors, ${ }^{13} 21242532$ we expect that the highest co-occurrence rates can be found for psychosis, unipolar depressive disorders, social anxiety disorder, schizoid personality disorder, avoidant personality disorder, post-traumatic stress disorder, and Internet/game addiction.

\section{METHODS}

The current protocol was registered in PROSPERO on CRD 42018098747 and reported in accordance with the criteria of the PRISMA-protocol (PRISMA-P). ${ }^{41}$ Any amendments will be updated on PROSPERO and documented accordingly.

\section{Eligibility criteria}

According to the PRISMA guidelines, the criteria considered for inclusion of the studies will involve participants, outcomes and research design. Studies will be included if: (a) they are conducted on young individuals aged 12-35 recruited from the general population (community or student/undergraduate population) or from treatment-seeking clinical populations with psychiatric disorders, diagnosed according to the criteria of international classification systems (eg, DSM- 5$)^{42}$ referred to primary, secondary or tertiary mental health settings, (b) they report the data necessary to calculate the effect sizes as event rates on point, period or lifetime prevalence of HS (sample size of the total sample, number of participants reporting the HS and number of participants with a specific psychiatric disorder according to a classification system) and the study authors are available to provide the necessary data when they are contacted, if such data are missing in the paper, (c) they are based on observational cross-sectional, case-control or longitudinal research designs, (d) they use self-report instruments, clinician-administered interviews or proxy-reported questionnaires to assess the HS, (e) they have been conducted on and have recruited the participants in the general population, high-schools, universities or in clinical settings, including primary, secondary or tertiary healthcare settings, (f) they have been published in peer-reviewed journals, (g) they investigated the HS and conceptualised it as an independent psycho-sociological condition 
including the following features: (1) spending most of the day and almost every day at home, (2) marked and persistent avoidance of social situations, (3) the social withdrawal and avoidance interferes significantly with the person's normal routine, occupational (or academic) functioning, or social activities or relationships, (4) the person perceives the withdrawal as ego-syntonic, (5) in individuals under age 18 years, the duration is at least 6 months, (6) the social withdrawal is independent from and not better accounted for by another mental disorder (eg, social anxiety disorder, major depressive disorder, schizophrenia or avoidant personality disorder). Despite numerous criteria were proposed in the literature to define the HS including those suggested by Kim $e t a l^{43}$ by Tateno $e t a l^{44}$ or the criteria by the Japanese Ministry of Health, Labour and Welfare published in $2010,{ }^{45}$ we have used the above-mentioned criteria following the definition provided by Teo and $\mathrm{Gaw}^{13}$ who identified the most recurrent clinical features in papers published in peer-reviewed journals. The presence of a concurrent treatment, either pharmacological or psychological, will not be an exclusion criterion. Studies conducted on the efficacy of a treatment will be included, if they report the necessary data to calculate the prevalence rates or the authors agree to provide such data for the systematic review purposes (see point ' $b$ ' of this paragraph and 'Meta-analysis' paragraph). No restriction regarding language and publication date will be applied.

Studies will be excluded if they conceptualise the HS as a symptom accounted for by another major psychiatric disorder defined by international classification systems (eg, DSM-5) such as psychotic/bipolar disorders, depressive disorders, Internet addiction, social anxiety disorder or a personality disorder (eg, schizoid or avoidant personality disorder). Reviews, case reports, case series, opinion papers, and anecdotal reports will be excluded. Studies will be excluded if they have been conducted using participants who present with mental retardation, neurological disorders or any medical disorder that implies a physical disability. No publication date restriction will be applied.

\section{Information sources and search procedure}

A detailed overview of the full search strategy is displayed in the online supplementary material. Published studies will be identified by conducting an online systematic search of electronic databases. The PubMed Medical Subject Headings (MeSH) on Demand Tool allowed us to generate relevant MeSH terms. MeSH terms, text words or title/abstract words related to the HS concept ('Social withdrawal' OR 'Hikikomori Syndrome' OR 'Hidden youth' OR 'Severe social isolation' OR 'Socially withdrawn youth' OR 'Young hermits') will be used alone. In addition, they will be combined with MeSH terms, text words or title/abstract words related to major psychiatric disorders ('psychiatric disorder' OR 'personality disorder' OR 'psychosis' OR 'bipolar disorder' OR 'depressive disorder' OR 'social anxiety disorder' OR 'post-traumatic stress disorder' OR 'obsessive compulsive disorder' OR 'generalised anxiety disorder' OR 'panic disorder' or 'agoraphobia' OR 'eating disorder' OR 'substance addiction' OR 'alcohol addiction' OR 'Internet addiction' OR 'game addiction') through the boolean operator AND. The search procedure will be conducted during the first week of November 2019 (start date: 4 November 2019; end date: 11 November 2019), using the databases Scopus, PubMed, PsycINFO and Web of Science. This search strategy will be used for each one of the databases. In addition, to identify further studies, all the corresponding authors of the studies to be included will be contacted by e-mail. The reference section of each of the studies included will be examined. In addition, the references of previous reviews will be examined to search additional studies to be included. ${ }^{12}$ 32 34-36 4647 Conference proceedings, abstract books and posters will be hand-searched in the following international scientific societies relevant to the topic: World Psychiatry Association, American Psychiatry Association, American Psychological Association, European Association of Psychiatry, European Association of Psychology, British Psychological Society, Royal College of Psychiatrists, World Association of Social Psychiatry, European Society of Social Psychiatry, American Association of Social Psychiatry. Finally, a further online search will be conducted to identify thesis/doctoral dissertations. They will be identified by the two independent reviewers who will run the same queries on Open Access Theses and Dissertations website.

\section{Selection of the studies}

Studies will be assessed and screened on eligibility criteria by two independent reviewers in three stages (AP, FF). During the first and second stages, studies will be assessed with regard to inclusion criteria after reading the title, then the abstract, respectively. During the selection based on the title, duplicates will be removed. During the selection based on the title or the abstract, studies on irrelevant constructs will be excluded. Studies will be classified as related to irrelevant constructs at title or abstract if they do not focus on the HS or prolonged social withdrawal in youth. After each stage, the reviewers will meet to compare their selections. Studies will be retained if there is not agreement between the reviewers on inclusion or exclusion. During the final stage, studies will be assessed independently by the two reviewers examining the full text of the paper. Potential discrepancies on inclusion or exclusion at this stage and their reasons will be discussed and resolved in a meeting with two independent reviewers (AC, MG) to reach consensus and to obtain a shared number of included studies. A third reviewer (AC) will assess independently the paper on inclusion/exclusion criteria and, finally, during a meeting between the first three reviewers and the fourth reviewer (MG) the decision whether the paper should be included or not will be reached by consensus. Between-reviewer agreement on inclusion will be calculated by Kappa index. ${ }^{48}$ 


\section{Data extraction and coding procedure}

All the information will be extracted from each of the included studies and inserted in an Excel worksheet by two independent reviewers (AP, FF) who will develop and pilot it first on two included studies, randomly extracted by the total group of the included primary studies. The following information will be extracted and coded from each of the included studies: (1) title of the paper, (2) first author, (3) publication date, (4) country where the study has been conducted (coded as Asian or non-Asian country), (5) inclusion and exclusion criteria, (6) total sample size, (7) number of participants reporting the HS according to the above-mentioned criteria, (8) criteria used to define the HS, (9) number of participants reporting a specific psychiatric disorder, (10) mean age of the total sample in the study, (11) percentage of females of the total sample in the study, (12) research design, (13) name of the instrument(s) used to assess the HS, (14) type of the instrument(s) used to assess the HS (self-report questionnaire, clinician-administered interview, proxy-reported questionnaire, proxy-reported interview), (15) type of population where the study sample has been drawn (general population, undergraduates, high-school students, clinical sample with a psychiatric disorder), (16) setting where the participants have been recruited, (17) strategies used to recruit the patients. The two independent reviewers will code the psychiatric disorders by pooling them in broad diagnostic categories

Box 1 Coding of broad diagnostic psychiatric categories pooled across classification systems.

Psychosis/bipolar disorders and related disorders.

- (eg, schizoaffective disorder, schizophreniform disorder, psychotic episode).

- Unipolar depressive disorders and related mood disorders.

- (eg, single episode/recurrent depressive disorder, dysthymic disorder).

- Social anxiety disorder.

- Post-traumatic stress disorder and related disorders.

- Obsessive compulsive disorder and related disorders.

- (eg, hoarding disorder, body dysmorphic disorder, skin picking disorder/trichotillomania).

- Generalised anxiety disorder.

- Panic disorder/agoraphobia.

- Eating disorders (eg, anorexia nervosa, bulimia nervosa, binge eating disorder).

- Substance/alcohol/drug addiction.

- Internet/game addiction.

- Schizoid/schizotypal personality disorder.

- Paranoid personality disorder.

- Antisocial personality disorder.

Borderline personality disorder.

- Hystrionic personality disorder.

- Narcissistic personality disorder.

- Obsessive compulsive personality disorder.

- Avoidant personality disorder.

Dependent personality disorder. across different versions of the classification systems. The coding system is displayed in box 1 .

Two independent reviewers (AC, MG), not involved in the extraction procedure, will check independently the correctness of the data inserted in the worksheet and the coding procedure. After the insertion of the data is conducted, potential discrepancies in the data extracted by the two reviewers will be discussed in a staff meeting between the reviewers who conduct the data extraction and the two independent reviewers who check the procedure.

\section{Quality assessment}

Quality of the included studies will be evaluated by using the Newcastle-Ottawa Scale (NOS) ${ }^{49}$ This tool has been recently recommended by systematic review practice guidelines as the most reliable instrument for conducting quality assessment of cross-sectional or cohort studies in systematic reviews. ${ }^{50}$ The NOS includes eight items, grouped into three key domains: (1) selection, (2) comparability, (3) outcome (cohort studies) or exposure (case-control studies) according to the study design. For each item a series of response options is provided. A star system is adopted to allow a semi-quantitative quality assessment: the highest quality studies obtain a maximum of one star for each item, apart from the item related to comparability, that allows the assignment of two stars. The scores on the NOS range from zero to nine stars. Two independent reviewers (AP, FF) will conduct the quality assessment. Potential discrepancies will be resolved by a consensus meeting with other two independent reviewers $(\mathrm{MG}, \mathrm{AC})$.

\section{Meta-analysis}

A random-effect meta-analysis will be conducted through the Software Comprehensive Meta-Analysis, CMA V.2.00. Random-effect models assume that the included studies are drawn from populations of studies that systematically differ from each other. ${ }^{51}$ According to these models, the effect sizes extracted from the included studies differ not only because of the random error within studies (as in the fixed-effects model) but also because of true variation in effect sizes from one study to the other.

The effect sizes on the worldwide prevalence rates will be calculated as event rates (ie, the ratio between the number of cases with HS and the total sample size). Co-occurrence between HS and a specific psychiatric disorder will be evaluated through two types of effect sizes calculated for each psychiatric disorder defined according to any DSM or ICD version: (1) the ratio between the number of cases with a specific psychiatric disorder and the total sample size of individuals reporting HS, (2) the ratio between the number of cases with HS and the total sample size of individuals with a specific psychiatric disorder. All effect sizes expressed will be transformed in logit event rates: higher effect sizes indicate higher prevalence rates of the HS. The effect sizes will be estimated using a 99\% CI and interpreted according to criteria proposed by $\operatorname{Cohen}^{52}$ : 
values equal to 0.80 or higher will be interpreted as large, up to 0.50 as moderate and up to 0.20 as small. Since the meta-analysis investigates the prevalence rates of the HS in studies conducted in general population and in studies conducted on clinical samples, two separate meta-analyses will be performed by calculating the effect sizes following the above-mentioned procedure.

Heterogeneity analysis will be conducted by calculating the $\mathrm{I}^{2}$ statistic ${ }^{53}$ and the $Q$ index. ${ }^{54}$ The $\mathrm{I}^{2}$ index represents a measure of variation in percentage across studies, that is attributable to heterogeneity rather than chance. ${ }^{53}$ A value approximating to zero suggests homogeneity, whereas values of $25 \%-50 \%, 50 \%-75 \%$ and $75 \%-100 \%$ represent low, medium and large heterogeneities, respectively. The $Q$ index is calculated by summing the squared deviations of each study's effect estimate from the overall effect estimate, while weighting the contribution of each study by its inverse variance. ${ }^{54}$ Under the hypothesis of homogeneity among the effect sizes, the $Q$ statistic follows a $\chi^{2}$ distribution with $k-1$ degrees of freedom, $k$ being the number of studies.

Since significant heterogeneity is expected, gender and age will be investigated as moderators of the effect sizes by calculating weighted least squares meta-regressions. In addition, potential differences as a function of type of country will be assessed by calculating mixed-model Analysis of variance (ANOVA).

To assess the likelihood that the effect sizes are subjected to publication bias, two procedures will be used: the Duval and Tweedie's trim and fill procedure and the visual inspection of the funnel plot. ${ }^{51}$ The funnel plot represents a scatter plot in which the effect sizes computed from the included studies are plotted on the horizontal axis against an indicator of study precision, the standardised error, on the vertical axis. ${ }^{53}$ In the absence of bias, the graph resembles a symmetrical inverted funnel, because the effect sizes derived from smaller studies scatter more widely at the bottom of the graph, with the spread narrowing with increasing precision among larger studies. If there is publication bias because smaller studies that report no significant effect sizes are not published, then the funnel plot appears asymmetrical. ${ }^{53}$

\section{Patient and public involvement}

Patients and the public were not involved in the development phase of the research question, of the outcome measures and of the systematic review and meta-analysis protocol. The study does not involve patient recruitment, and patients were not involved in conduct of the study. The results will be disseminated through conference presentations and publications in peer-reviewed journals.

\section{Discussion and conclusions}

The HS is a psycho-sociological condition, which has increasingly drawn the attention of researchers and practitioners working in the mental health field. ${ }^{3-5} 13$ Despite the growing number of studies in the literature, a systematic review with meta-analysis is lacking to summarise the data on the prevalence rates of the HS in the general population and in the clinical population with psychiatric disorders. Providing a quantitative synthesis can allow the limitations in the current literature to be identified and further research to suggest what should be investigated. This protocol describes the methodology of a systematic review and meta-analysis which will examine the worldwide prevalence rates of the HS in general and in clinical population. The review will aim to assess the co-occurrence between HS and each psychiatric disorder defined according to the criteria of a classification system. The potential strengths of the study will be that the previous review $^{12}$ conducted the online search in February 2015, did not carry out a meta-analysis and did not examine the prevalence rates. Thus, a strength of this study will be that it may add more recent data, published in the last 4 years. In addition, at the present time no review has been conducted to summarise the co-occurrence rates of HS and psychiatric disorders by meta-analytic methods.

While some studies have been conducted in the general population, other research used clinical samples, leading to very different prevalence rates; thus, it could be important to identify the prevalence rates in the two different populations. Providing a quantitative summary could add knowledge to the understanding of the HS phenomenon and allow us to compare its prevalence with the data regarding other mental health disorders, which have some clinical overlap (eg, psychotic disorders, depressive disorders, personality disorders). In addition, a summary of the co-occurrence rates of each psychiatric disorder and HS may allow us to identify the psychiatric diagnoses most frequently associated with this condition.

In conclusion, given the increasing public and scientific awareness raised by the HS in the Japanese society but also around the world, the current systematic review could provide additional useful evidence for researchers, clinicians and policymakers on a still under-recognised social problem.

\section{ETHICS AND DISSEMINATION}

The current review protocol does not require ethics approval. The results will be disseminated through publication in peer-reviewed journals.

Contributors AP designed and conceived the study, wrote the first draft of the paper, will conduct the search, data screening, data extraction and coding. AC designed and conceived the study, critically reviewed the first draft of the paper, will check data screening, data extraction and coding. TK designed and conceived the study, critically reviewed the first draft of the paper, will check data screening. MG designed and conceived the study, critically reviewed the first draft of the paper, will check data screening, data extraction and coding. FF designed and conceived the study, wrote the first draft of the paper, will conduct the search, data screening, data extraction and coding.

Funding The authors have not declared a specific grant for this research from any funding agency in the public, commercial or not-for-profit sectors.

Competing interests None declared.

Patient consent for publication Not required.

Provenance and peer review Not commissioned; externally peer reviewed. 
Data availability statement № data are available.

Open access This is an open access article distributed in accordance with the Creative Commons Attribution Non Commercial (CC BY-NC 4.0) license, which permits others to distribute, remix, adapt, build upon this work non-commercially, and license their derivative works on different terms, provided the original work is properly cited, appropriate credit is given, any changes made indicated, and the use is non-commercial. See: http://creativecommons.org/licenses/by-nc/4.0/.

\section{REFERENCES}

1. Furuhashi T, Tsuda H, Ogawa T, et al. État des lieux, points communs et différences entre des jeunes adultes retirants sociaux en France et Au Japon (Hikikomori). L'Evolution Psychiatrique 2013;8:249-66.

2. Saito T, Angles J. Hikikomori: adolescence without end. Minneapolis: University of Minnesota Press, 2013.

3. Kaneko S. Japan's 'socially withdrawn youths' and time constraints in Japanese society: management and conceptualization of time in a support group for 'hikikomori'. Time \& Society 2006;5:233-49.

4. Saito T. Shakaiteki hikikomori - owaranai shishunki [Social hikikomori - Neverending Adolescence. Tokyo: PHP Shinsho, 1998.

5. Watts J. tokyo Public health experts concerned about "hikikomori". Lancet 2002;359.

6. Kondo N, Sakai M, Kuroda Y, et al. General condition of hikikomori (prolonged social withdrawal) in Japan: Psychiatric diagnosis and outcome in mental health welfare centres. Int J Soc Psychiatry 2013;59:79-86.

7. Ito J, Yoshida M, Kobayashi K. Report of investigation into the actual condition among consultations about social withdrawal (hikikomori). Guideline from the Ministry of Health and Labor, 2003.

8. Ministry of Health, Labour \& Welfare. Community mental health intervention guidelines aimed at socially withdrawn teenagers and young adults. Tokyo: Ministry of Health, Labour \& Welfare, 2003.

9. Todd KHL. Hikikomania: Existential horror or national malaise. Southeast Review of Asian Studies 2011;33:135-47.

10. Heinze U, Thomas P. Self and salvation: visions of hikikomori in Japanese manga. D / J 2014;26:151-69.

11. Kato TA, Shinfuku N, Sartorius N, et al. "Kodoku-shi and Hikikomori" in Loneliness and Single-Person Households: Issues of KodokuShi and Hikikomori in Japan. In: Okkels N, Blanner Kristiansen C, Munk-Jorgensen P, et al, eds. Mental health and illness in the City. Singapore: Springer, 2017: 1-13.

12. TM L, Wong PW. Youth social withdrawal behavior (hikikomori): a systematic review of qualitative and quantitative studies. Aust N Z J 2015;49:595-609.

13. Teo AR, Gaw AC. Hikikomori, a Japanese culture-bound syndrome of social withdrawal?: a proposal for DSM-5. J Nerv Ment Dis 2010;198:444-9.

14. Coluccia A, Fagiolini A, Ferretti F, et al. Obsessive-Compulsive disorder and quality of life outcomes: protocol for a systematic review and meta-analysis of cross-sectional case-control studies. Epidemiol, Biostat Public Health 2015;12.

15. Coluccia A, Ferretti F, Fagiolini A, et al. Incidenza E fattori di rischio per disturbi psicotici nelle popolazioni migranti in Europa: Una meta-analisi di studi trasversali. Rassegna Italiana di Criminologia 2015;9:29-39.

16. Coluccia A, Ferretti F, Fagiolini A, et al. Quality of life in children and adolescents with obsessive-compulsive disorder: a systematic review and meta-analysis. Neuropsychiatr Dis Treat 2017;13:597-608.

17. Dèttore D, Berardi D, Pozza A. Religious affiliation and obsessive cognitions and symptoms: a comparison between Jews, Christians, and Muslims in non-clinical groups in Italy. Psychol Relig Spiritual 2017:9:348-57.

18. Hofmann SG, Anu Asnaani MA, Hinton DE. Cultural aspects in social anxiety and social anxiety disorder. Depress Anxiety 2010;27:1117-27.

19. Parker G, Cheah Y-C, Roy K. Do the Chinese somatize depression? A cross-cultural study. Soc Psychiatry Psychiatr Epidemiol 2001;36:287-93.

20. Pozza A, Dèttore D. Are inflated responsibility beliefs specific to OCD? meta-analysis of the relations of responsibility to OCD, anxiety disorders, and depression symptoms. Clin Neuropsychiatry 2014;11:170-81.

21. Teo AR, Fetters MD, Stufflebam K, et al. Identification of the hikikomori syndrome of social withdrawal: psychosocial features and treatment preferences in four countries. Int $J$ Soc Psychiatry 2015;61:64-72.
22. Kato TA, Tateno M, Shinfuku N, et al. Does the 'hikikomori' syndrome of social withdrawal exist outside Japan? A preliminary international investigation. Soc Psychiatry Psychiatr Epidemiol 2012;47:1061-75.

23. Kondo N, Miyazawa H, Sakai M, et al. Shishunki hikikomori Ni okeru seishin igakuteki shougai no jittai haaku Ni kan suru kenkyuu (research on the psychiatric disorders in adolescents with social withdrawal). Kokoro no kenkou kagaku kenkyuu 2008:49-63.

24. Kato TA, Kanba S, Teo AR. Hikikomori: multidimensional understanding, assessment and future international perspectives. Psychiatry Clin Neurosci 2019:427-40.

25. Furlong $A$. The Japanese hikikomori phenomenon: acute social withdrawal among young people. Sociol Rev 2008;56:309-25.

26. Koyama A, Miyake Y, Kawakami N, et al. Lifetime prevalence, psychiatric comorbidity and demographic correlates of "hikikomori" in a community population in Japan. Psychiatry Res 2010;176:69-74

27. Wong PWC, Li TMH, Chan M, et al. The prevalence and correlates of severe social withdrawal (hikikomori) in Hong Kong: A cross-sectional telephone-based survey study. Int J Soc Psychiatry 2015;61:330-42.

28. Lee YS, Lee JY, Choi TY, et al. Home visitation program for detecting, evaluating and treating socially withdrawn youth in Korea. Psychiatry Clin Neurosci 2013;67:193-202.

29. Norasakkunkit V, Uchida Y. To Conform or to Maintain SelfConsistency? Hikikomori Risk in Japan and the Deviation From Seeking Harmony. J Soc Clin Psychol 2014;33:918-35.

30. Á M-A, Córcoles-Martínez D, Martín-López LM, et al. Hikikomori in Spain: a descriptive study. Int J Soc Psychiatry 2015;61:475-83.

31. Wong V. Youth locked in time and space? defining features of social withdrawal and practice implications. J Soc Work Pract 2009;23:337-52.

32. Chan H-yee, Lo T-wing. Quality of life of the hidden youth in Hong Kong. Appl Res Qual Life 2014;9:951-69.

33. Kato TA, Kanba S, Teo AR. Hikikomori: experience in Japan and international relevance. World Psychiatry 2018;17:105-6.

34. Kiyota A, Usami M, Oosumi H. Chiiki renkei shisutemu N yoru hikikomori shien to ekigakuteki kentou (support and epidemiological analysis of social withdrawal using a system of regional parternships). Kokoro no kenkou kagaku kenkyuu 2008:95-101.

35. Stip E, Thibault A, Beauchamp-Chatel A, et al. Internet addiction, hikikomori syndrome, and the prodromal phase of psychosis. Front. Psychiatry 2016;7.

36. Tajan N. Social withdrawal and psychiatry: a comprehensive review of Hikikomori. Neuropsychiatr Enfance Adolesc 2015;63:324-31.

37. Moher D, Liberati A, Tetzlaff J, et al. Preferred reporting items for systematic reviews and meta-analyses: the PRISMA statement. PLoS Med 2009;6:e1000097.

38. Hattori Y. Social withdrawal in Japanese youth: a case study of thirtyfive hikikomori clients. J Trauma Pract 2006;4:181-201.

39. Teo AR. Social isolation associated with depression: A case report of hikikomori. Int J Soc Psychiatry 2013;59:339-41.

40. Moher D, Shamseer L, Clarke M, et al. Preferred reporting items for systematic review and meta-analysis protocols (PRISMA-P) 2015 statement. Syst Rev 2015;4:1.

41. Shamseer L, Moher D, Clarke M, et al. Preferred reporting items for systematic review and meta-analysis protocols (PRISMA-P) 2015 elaboration and explanation. BMJ 2015;349:g7647.

42. American Psychiatric Association. Diagnostic and statistical manual of mental disorders. 5th ed. Washington, DC: American Psychiatric Association, 2013

43. Kim Y, Horiguchi I, Mori M. Tiiki Seishi Hoken Katsudo Ni Okeru Kainyuu No Arikata Ni Kansuru Kenkyuu [A preliminary study about prevalence of "hikikomori" in young adults]. In: Ito J, ed. Heisei Nen Soukatsu Houkokusyo (a study on intervention in community mental health Activity - 2001 research report). Ichikawa, Japan: National Institute of Mental Health, 2012.

44. Tateno M, Park TW, Kato TA, et al. Hikikomori as a possible clinical term in psychiatry: a questionnaire survey. BMC Psychiatry 2012:12:169.

45. Saito K. Hikikomori no hyouka: shien Ni kansuru gaidorain. Tokyo: Japan's Ministry of Health, Labour and Welfare, 2010.

46. Sarchione F, Santacroce R, Acciavatti T, et al. Hikikomori, clinical and psychopathological issues. Res Adv Psychiatry 2015;2:21-7.

47. Teo AR. A New Form of Social Withdrawal in Japan: a Review of Hikikomori. Int J Soc Psychiatry 2010;56:178-85.

48. Cohen J. A coefficient of agreement for nominal scales. Educ Psychol Meas 1960;20:37-46.

49. Wells G, Shea B, O'Connell D, et al. Newcastle-Ottawa quality assessment scale cohort studies, 2015. Available: http://www. ohri. ca/programs/clinical_epidemiology/oxford

50. Zeng X, Zhang Y, Kwong JSW, et al. The methodological quality assessment tools for preclinical and clinical studies, systematic 
review and meta-analysis, and clinical practice guideline: a systematic review. J Evid Based Med 2015;8:2-10.

51. Borenstein M, Hedges LV, Higgins JPT, et al. Introduction to metaanalysis. Chichester: John Wiley \& Sons, 2009.

52. Cohen J. Statistical power analysis for the behavioral sciences. New York: Routledge, 1988.
53. Higgins JPT, Thompson SG. Quantifying heterogeneity in a metaanalysis. Stat Med 2002;21:1539-58.

54. Hedges LV, Vevea JL. Fixed- and random-effects models in metaanalysis. Psychol Methods 1998;3:486-504. 